\title{
Existence of solutions for tripled system of fractional differential equations involving cyclic permutation boundary conditions
}

\author{
Mohammed M. Matar ${ }^{1}$, Iman Abo Amra ${ }^{1}$ and Jehad Alzabut ${ }^{2 *}$ (1)
}

\section{"Correspondence:}

jalzabut@psu.edu.sa

${ }^{2}$ Department of Mathematics and

General Sciences, Prince Sultan

University, Riyadh, 11586, Saudi

Arabia

Full list of author information is

available at the end of the article

\begin{abstract}
In this paper, we introduce and study a tripled system of three associated fractional differential equations. Prior to proceeding to the main results, the proposed system is converted into an equivalent integral form by the help of fractional calculus. Our approach is based on using the addressed tripled system with cyclic permutation boundary conditions. The existence and uniqueness of solutions are investigated. We employ the Banach and Krasnoselskii fixed point theorems to prove our main results. Illustrative examples are presented to explain the theoretical results.
\end{abstract}

MSC: $34 \mathrm{~A} 08 ; 34 \mathrm{~A} 128$

Keywords: Tripled fractional differential system; Cyclic permutation; Tripled boundary conditions; Banach and Krasnoselskii fixed point theorems

\section{Introduction}

Fractional derivatives and integrals find numerous applications in many branches of physics and engineering ranging from quantum optics to astro-physics and cosmology, dynamics of materials to biophysics and medicine, dynamical chaos to control, signal processing to communications, and more. For recent comprehensive reviews on fractional derivatives and their applications, we refer the reader to the monographs [25, 34, 36] and the recent undermentioned papers $[1-3,5,6,9,11,13,17,23,26-29,31,32,35]$. Due to their widespread applications, a system of fractional differential equations subject to boundary conditions has received much attention amongst researchers who accommodate various numerical methods to establish their results; see for instance the papers $[18,22,33]$.

Particularly, coupled fractional boundary systems, which study interaction between two quantities, have been under consideration as they provide adequate interpretations for models describing chaotic behavior, anomalous diffusion, ecological effects, and biological models. Many relevant results have been reported in this direction with different boundary conditions; see $[4,7,8,14-16,21,30,37-39]$ and the references therein.

Tripled fractional boundary systems, which are considered as a generalization of coupled fractional systems, are governed by three associated differential equations with three

(c) The Author(s) 2020. This article is licensed under a Creative Commons Attribution 4.0 International License, which permits use, sharing, adaptation, distribution and reproduction in any medium or format, as long as you give appropriate credit to the original author(s) and the source, provide a link to the Creative Commons licence, and indicate if changes were made. The images or other third party material in this article are included in the article's Creative Commons licence, unless indicated otherwise in a credit line to the material. If material is not included in the article's Creative Commons licence and your intended use is not permitted by statutory regulation or exceeds the permitted use, you will need to obtain permission directly from the copyright holder. To view a copy of this licence, visit http://creativecommons.org/licenses/by/4.0/. 
initial or boundary conditions [12, 24]. In [12], Berinde and Borcut introduced the concept of tripled fixed point for nonlinear mappings in partially ordered complete metric spaces and obtained existence and uniqueness theorems for contractive type mappings. Karakaya et al.[24] gave some results concerning the existence of tripled fixed points for a class of condensing operators in Banach spaces.

The cyclic boundary conditions have many applications on channel flow with fully developed flow at inlet as well as outlet using simple foam. In addition, some researchers introduced a railway track coupled dynamics model based on cyclic boundary conditions (see [10] and the references therein).

Unlike coupled fractional systems, the investigations of tripled fractional systems have gained less attention amongst researchers. To the best of authors' observation, indeed, there is no analytical literature on studying the existence of tripled systems of fractional differential equations.

Motivated by these research works, we investigate in this paper a tripled fractional abstract system with cyclic tripled boundary conditions that has the following form:

$$
\begin{cases}{ }^{c} D_{0}^{\alpha_{k}} x_{k}(t)=f_{k}(t, x(t)), & 1<\alpha_{k} \leq 2, \\ x_{k}^{(j)}(0)=a_{k, j} x_{\sigma(k)}^{(j)}(T), & k=1,2,3 ; j=0,1,\end{cases}
$$

where ${ }^{c} D_{0}^{\alpha_{k}}$ denotes the Caputo fractional derivative of order $\alpha_{k}, t \in J=[0, T], f_{k}: J \times \mathbb{R}^{3} \rightarrow$ $\mathbb{R}$ are continuous functions, $x=\left(x_{1}, x_{2}, x_{3}\right) \in \mathbb{R}^{3}, \sigma=\left(\begin{array}{lll}1 & 2 & 3\end{array}\right)$ is a cycle permutation, and $a_{k, j} \in \mathbb{R}, k=1,2,3, j=0,1$, such that $\prod_{k=1}^{3} a_{k, j} \neq 1, j=0,1$. System (1.1) is converted into an equivalent integral form by the help of fractional calculus. The existence and uniqueness of solutions with cyclic permutation of tripled boundary conditions are investigated. We employ the Banach and Krasnoselskii fixed point theorems to prove our main results.

The railway track coupled system investigated in [10] can be modeled as a classical tripled system if it undergoes an external influence, by which many researchers can be prompted to generalize this idea using fractional differential models. We emphasize that the problem considered in the present settings is new and has novel approach that will provide further insight into the analytical study of tripled fractional systems with cyclic boundary conditions.

\section{Preliminary assertions}

In this section, we recall some basic definitions of fractional calculus [25]. Meanwhile, the integral form of the solution of system (1.1) as well as the definition of permutation group are presented. The notations and terminologies herein will be used in the subsequent section.

Definition 2.1 ([25]) The Riemann-Liouville fractional integral of a real-valued function $f \in C(J)$ is defined by

$$
I_{0}^{\alpha} f(t)=\int_{0}^{t} \frac{(t-s)^{\alpha-1}}{\Gamma(\alpha)} f(s) d s, \quad t \in J, \alpha>0,
$$


provided the integral exists, and $I_{0}^{0} f(t):=f(t)$. The Caputo fractional derivative of $f \in$ $C^{(n)}(J)$ is given by

$$
{ }^{c} D_{0}^{\alpha} f(t)=I_{0}^{n-\alpha} f^{(n)}(t),
$$

where $n=[\alpha]$ is the greatest integer function.

Lemma $2.2([25])$ Let $[\alpha]=n \in \mathbb{N}$, and $f,{ }^{c} D_{0}^{\alpha} f \in C(J)$. Then

$$
I_{0}^{\alpha c} D_{0}^{\alpha} f(t)=f(t)+c_{0}+c_{1} t+c_{2} t^{2}+\cdots+c_{n-1} t^{n-1}
$$

for $c_{i} \in \mathbb{R}, i=0,1,2, \ldots, n-1$.

For convenience, we introduce the following notations:

$$
\begin{aligned}
& b_{1,1}=b_{2,2}=b_{3,3}=\frac{a_{1,1} a_{2,1} a_{3,1}}{1-a_{1,1} a_{2,1} a_{3,1}}, \quad b_{1,2}=\frac{a_{1,1}}{1-a_{1,1} a_{2,1} a_{3,1}}, \\
& b_{1,3}=\frac{a_{1,1} a_{2,1}}{1-a_{1,1} a_{2,1} a_{3,1}}, \quad b_{2,1}=\frac{a_{2,1} a_{3,1}}{1-a_{1,1} a_{2,1} a_{3,1}}, \quad b_{2,3}=\frac{a_{2,1}}{1-a_{1,1} a_{2,1} a_{3,1}}, \\
& b_{3,1}=\frac{a_{3,1}}{1-a_{1,1} a_{2,1} a_{3,1}}, \quad b_{3,2}=\frac{a_{3,1} a_{1,1}}{1-a_{1,1} a_{2,1} a_{3,1}}, \\
& d_{1,1}=d_{2,2}=d_{3,3}=\frac{a_{1,0} a_{2,0} a_{3,0}}{1-a_{1,0} a_{2,0} a_{3,0}}, \quad d_{1,2}=\frac{a_{1,0}}{1-a_{1,0} a_{2,0} a_{3,0}}, \\
& d_{1,3}=\frac{a_{1,0} a_{2,0}}{1-a_{1,0} a_{2,0} a_{3,0}}, \quad d_{2,1}=\frac{a_{2,0} a_{3,0}}{1-a_{1,0} a_{2,0} a_{3,0}}, \quad d_{2,3}=\frac{a_{2,0}}{1-a_{1,0} a_{2,0} a_{3,0}}, \\
& d_{3,1}=\frac{a_{3,0}}{1-a_{1,0} a_{2,0} a_{3,0}}, \quad d_{3,2}=\frac{a_{3,0} a_{1,0}}{1-a_{1,0} a_{2,0} a_{3,0}}, \\
& e_{1,1}=\frac{a_{1,0} a_{3,1} T\left(a_{2,0} a_{3,0} a_{1,1} a_{2,1}+a_{2,0}+a_{2,1}\right)}{\left(1-a_{1,0} a_{2,0} a_{3,0}\right)\left(1-a_{1,1} a_{2,1} a_{3,1}\right)} \text {, } \\
& e_{1,2}=\frac{a_{1,0} a_{1,1} T\left(a_{2,0} a_{3,0}+a_{2,0} a_{3,1}+a_{2,1} a_{3,1}\right)}{\left(1-a_{1,0} a_{2,0} a_{3,0}\right)\left(1-a_{1,1} a_{2,1} a_{3,1}\right)}, \\
& e_{1,3}=\frac{a_{1,0} a_{2,1} T\left(a_{2,0} a_{3,0} a_{1,1}+a_{2,0} a_{1,1} a_{3,1}+1\right)}{\left(1-a_{1,0} a_{2,0} a_{3,0}\right)\left(1-a_{1,1} a_{2,1} a_{3,1}\right)}, \\
& e_{2,1}=\frac{a_{2,0} a_{3,1} T\left(a_{1,0} a_{2,1} a_{3,0}+a_{1,1} a_{2,1} a_{3,0}+1\right)}{\left(1-a_{1,0} a_{2,0} a_{3,0}\right)\left(1-a_{1,1} a_{2,1} a_{3,1}\right)}, \\
& e_{2,2}=\frac{a_{2,0} a_{1,1} T\left(a_{1,0} a_{3,0} a_{2,1} a_{3,1}+a_{3,0}+a_{3,1}\right)}{\left(1-a_{1,0} a_{2,0} a_{3,0}\right)\left(1-a_{1,1} a_{2,1} a_{3,1}\right)} \text {, } \\
& e_{2,3}=\frac{a_{2,0} a_{2,1} T\left(a_{1,0} a_{3,0}+a_{3,0} a_{1,1}+a_{3,1} a_{1,1}\right)}{\left(1-a_{1,0} a_{2,0} a_{3,0}\right)\left(1-a_{1,1} a_{2,1} a_{3,1}\right)}, \\
& e_{3,1}=\frac{a_{3,0} a_{3,1} T\left(a_{1,0} a_{2,0}+a_{1,0} a_{2,1}+a_{1,1} a_{2,1}\right)}{\left(1-a_{1,0} a_{2,0} a_{3,0}\right)\left(1-a_{1,1} a_{2,1} a_{3,1}\right)} \text {, } \\
& e_{3,2}=\frac{a_{3,0} a_{1,1} T\left(a_{1,0} a_{2,0} a_{3,1}+a_{1,0} a_{2,1} a_{3,1}+1\right)}{\left(1-a_{1,0} a_{2,0} a_{3,0}\right)\left(1-a_{1,1} a_{2,1} a_{3,1}\right)}, \\
& e_{3,3}=\frac{a_{3,0} a_{2,1} T\left(a_{1,0} a_{2,0} a_{1,1} a_{3,1}+a_{1,0}+a_{1,1}\right)}{\left(1-a_{1,0} a_{2,0} a_{3,0}\right)\left(1-a_{1,1} a_{2,1} a_{3,1}\right)} \text {. }
\end{aligned}
$$


Lemma 2.3 Let $f_{k} \in C(J, \mathbb{R})$ and $\prod_{k=1}^{3} a_{k, j} \neq 1, j=0,1$. Then the solution of the linear fractional differential system

$$
{ }^{c} D_{0}^{\alpha_{k}} x(t)=f_{k}(t), \quad 1<\alpha_{k} \leq 2, t \in(0, T),
$$

subject to the conditions

$$
x_{k}^{(j)}(0)=a_{k, j} x_{\sigma(k)}^{(j)}(T), \quad k=1,2,3, j=0,1,
$$

is given by

$$
x_{k}(t)=\sum_{m=1}^{3}\left(d_{k, m} I_{0}^{\alpha_{m}} f_{m}(T)+\left(e_{k, m}+t b_{k, m}\right) I_{0}^{\alpha_{m}-1} f_{m}(T)\right)+I_{0}^{\alpha_{k}} f_{k}(t) .
$$

Proof Applying the fractional integral to both sides of (2.1) and using Lemma 2.2, we obtain

$$
x_{k}(t)=c_{k, 0}+c_{k, 1} t+I_{0}^{\alpha_{k}} f_{k}(t) .
$$

Hence, we deduce that

$$
x_{k}^{\prime}(t)=c_{k, 1}+I_{0}^{\alpha_{k}-1} f_{k}(t) .
$$

The boundary conditions in (2.2) imply that

$$
\left\{\begin{array}{l}
c_{1,0}=a_{1,0}\left(c_{2,0}+c_{2,1} T+I_{0}^{\alpha_{2}} f_{2}(T)\right), \\
c_{2,0}=a_{2,0}\left(c_{3,0}+c_{3,1} T+I_{0}^{\alpha_{3}} f_{3}(T)\right), \\
c_{3,0}=a_{3,0}\left(c_{1,0}+c_{1,1} T+I_{0}^{\alpha_{1}} f_{1}(T)\right),
\end{array}\right.
$$

and

$$
\left\{\begin{array}{l}
c_{1,1}=a_{1,1}\left(c_{2,1}+I_{0}^{\alpha_{2}-1} f_{2}(T)\right), \\
c_{2,1}=a_{2,1}\left(c_{3,1}+I_{0}^{\alpha_{3}-1} f_{3}(T)\right), \\
c_{3,1}=a_{3,1}\left(c_{1,1}+I_{0}^{\alpha_{1}-1} f_{1}(T)\right) .
\end{array}\right.
$$

By direct substitutions of the equations in (2.5), we get

$$
\begin{aligned}
& c_{1,1}=b_{1,1} I_{0}^{\alpha_{1}-1} f_{1}(T)+b_{1,2} I_{0}^{\alpha_{2}-1} f_{2}(T)+b_{1,3} I_{0}^{\alpha_{3}-1} f_{3}(T), \\
& c_{2,1}=b_{2,1} I_{0}^{\alpha_{1}-1} f_{1}(T)+b_{2,2} I_{0}^{\alpha_{2}-1} f_{2}(T)+b_{2,3} I_{0}^{\alpha_{3}-1} f_{3}(T),
\end{aligned}
$$

and

$$
c_{3,1}=b_{3,1} I_{0}^{\alpha_{1}-1} f_{1}(T)+b_{3,2} I_{0}^{\alpha_{2}-1} f_{2}(T)+b_{3,3} I_{0}^{\alpha_{3}-1} f_{3}(T) .
$$

Similarly, the equations in (2.6) together with the last constants lead to

$$
c_{1,0}=d_{1,1} I_{0}^{\alpha_{1}} f_{1}(T)+d_{1,2} I_{0}^{\alpha_{2}} f_{2}(T)+d_{1,3} I_{0}^{\alpha_{3}} f_{3}(T)
$$




$$
\begin{aligned}
& +e_{1,1} I_{0}^{\alpha_{1}-1} f_{1}(T)+e_{1,2} I_{0}^{\alpha_{2}-1} f_{2}(T)+e_{1,3} I_{0}^{\alpha_{3}-1} f_{3}(T), \\
c_{2,0}= & d_{2,1} I_{0}^{\alpha_{1}} f_{1}(T)+d_{2,2} I_{0}^{\alpha_{2}} f_{2}(T)+d_{2,3} I_{0}^{\alpha_{3}} f_{3}(T) \\
& +e_{2,1} I_{0}^{\alpha_{1}-1} f_{1}(T)+e_{2,2} I_{0}^{\alpha_{2}-1} f_{2}(T)+e_{2,3} I_{0}^{\alpha_{3}-1} f_{3}(T),
\end{aligned}
$$

and

$$
\begin{aligned}
c_{3,0}= & d_{3,1} I_{0}^{\alpha_{1}} f_{1}(T)+d_{3,2} I_{0}^{\alpha_{2}} f_{2}(T)+d_{3,3} I_{0}^{\alpha_{3}} f_{3}(T) \\
& +e_{3,1} I_{0}^{\alpha_{1}-1} f_{1}(T)+e_{3,2} I_{0}^{\alpha_{2}-1} f_{2}(T)+e_{3,3} I_{0}^{\alpha_{3}-1} f_{3}(T) .
\end{aligned}
$$

Substituting the values of $c_{k, j}, k=1,2,3, j=0,1$, in (2.4), we get (2.3). This completes the proof.

We adopt the following definition of permutation groups.

Definition 2.4 ([19]) A permutation of a set $A$ is a function $\sigma: A \rightarrow A$ that is one to one and onto.

This defines the so-called permutation group $(A, \sigma)$. Let $A=\{1,2,3\}$, then the cardinality of this group is $3 !=6$ permutations. For instance, one of such permutations is given by $\sigma(1)=2, \sigma(2)=3, \sigma(3)=1$, and this constitutes a cycle $\sigma=\left(\begin{array}{lll}1 & 2 & 3 \\ 2 & 3 & 1\end{array}\right)=\left(\begin{array}{lll}1 & 2 & 3\end{array}\right)$. However, we use this cycle in the boundary conditions of system (1.1) such that, for a triple $\left(x_{1}, x_{2}, x_{3}\right)$, we have $x_{1}^{(j)}(0)=a_{1, j} x_{2}^{(j)}(T), x_{2}^{(j)}(0)=a_{2, j} x_{3}^{(j)}(T)$, and $x_{3}^{j}(0)=a_{3, j} x_{1}^{j}(T), j=1,2$. The other five permutations can be used and another integral solution can be obtained which is isomorphic to the one in (2.3) with constant differences. To explain this more, we consider permutation (13)(2). As a consequence of Lemma 2.3, we find the same solution as (2.3) but with different coefficients. Indeed, we find the following:

$$
\begin{aligned}
& b_{1,1}=b_{3,3}=\frac{a_{1,1} a_{3,1}}{1-a_{1,1} a_{3,1}}, \quad b_{2,2}=\frac{a_{2,1}}{1-a_{2,1}}, \\
& b_{1,3}=\frac{a_{1,1}}{1-a_{1,1} a_{3,1}}, \quad b_{3,1}=\frac{a_{3,1}}{1-a_{3,1} a_{1,1}}, \\
& b_{1,2}=b_{2,1}=b_{2,3}=b_{3,2}=0, \\
& d_{1,1}=\frac{1}{1-a_{1,0} a_{3,0}}, \quad d_{2,2}=\frac{a_{2,0}}{1-a_{2,0}}, \quad d_{3,3}=\frac{a_{3,0} a_{1,0}}{1-a_{1,0} a_{3,0}}, \\
& d_{1,3}=\frac{a_{1,0}}{1-a_{1,0} a_{3,0}}, \quad d_{3,1}=a_{3,0}\left(\frac{2-a_{1,0} a_{3,0}}{1-a_{1,0} a_{3,0}}\right) \\
& d_{1,2}=d_{2,1}=d_{2,3}=d_{3,2}=0, \\
& e_{1,1}=a_{3,1} a_{1,0} T\left(\frac{1+a_{1,1} a_{3,0}}{\left(1-a_{1,0} a_{3,0}\right)\left(1-a_{1,1} a_{3,1}\right)}\right), \\
& e_{1,3}=a_{1,1} a_{1,0} T\left(\frac{a_{3,1}+a_{3,0}}{\left(1-a_{1,0} a_{3,0}\right)\left(1-a_{1,1} a_{3,1}\right)}\right), \\
& e_{2,2}=\frac{a_{2,1} a_{2,0} T}{\left(1-a_{2,0}\right)\left(1-a_{2,1}\right)}, \\
& e_{3,1}=a_{3,0} a_{3,1} T\left(\frac{a_{1,0}+a_{1,1}}{\left(1-a_{1,0} a_{3,0}\right)\left(1-a_{1,1} a_{3,1}\right)}\right),
\end{aligned}
$$




$$
\begin{aligned}
& e_{3,3}=a_{3,0} T\left(\frac{a_{3,1} a_{1,1} a_{1,0}}{\left(1-a_{1,0} a_{3,0}\right)\left(1-a_{3,1} a_{1,1}\right)}\right), \\
& e_{2,1}=0=e_{1,2}=e_{2,3}=e_{3,2}=0 .
\end{aligned}
$$

A tripled fixed point of a mapping is given next.

Definition 2.5 ([12]) An element $\left(x_{1}, x_{2}, x_{3}\right) \in X \times X \times X$ is called a tripled fixed point of a mapping $\digamma: X \times X \times X \rightarrow X$ if $\digamma\left(x_{1}, x_{2}, x_{3}\right)=x_{1}, \digamma\left(x_{2}, x_{1}, x_{3}\right)=x_{2}$, and $\digamma\left(x_{3}, x_{2}, x_{1}\right)=x_{3}$.

Define an operator $\Psi: X \times X \times X \rightarrow X \times X \times X$ such that

$$
\Psi\left(x_{1}, x_{2}, x_{3}\right)=\left(\digamma\left(x_{1}, x_{2}, x_{3}\right), \digamma\left(x_{2}, x_{1}, x_{3}\right), \digamma\left(x_{3}, x_{2}, x_{1}\right)\right) .
$$

Then $\left(x_{1}, x_{2}, x_{3}\right)$ is a tripled fixed point of $\digamma$ iff $\left(x_{1}, x_{2}, x_{3}\right)$ is a fixed point of $\Psi$, that is, $\Psi\left(x_{1}, x_{2}, x_{3}\right)=\left(x_{1}, x_{2}, x_{3}\right)$.

For completeness, we recall the following tools of fixed point theory.

Theorem 2.6 (Banach fixed point theorem [20]) Let D be a nonempty closed subset of a Banach space $E$. Then any contraction mapping $T$ from $D$ into itself has a unique fixed point.

Theorem 2.7 (Krasnoselskii fixed point theorem [20]) Let B be a closed convex and nonempty subset of a Banach space X. Let $\Psi_{1}, \Psi_{2}$ be operators defined on B such that

(i) $\Psi_{1} x+\Psi_{2} y \in B$ whenever $x, y \in B$;

(ii) $\Psi_{1}$ is a contraction mapping;

(iii) $\Psi_{2}$ is compact and continuous.

Then there exists $z \in B$ such that $z=\Psi_{1} z+\Psi_{2} z$.

\section{Main results}

In this section we use the Banach and Krasnoselskii fixed point theorems to ensure the existence of solution for tripled system (1.1).

The Banach space $X=C(J, \mathbb{R})$ of continuous real-valued functions is defined on $J$ with the usual maximum norm. Hence, we obtain a Banach space $X^{3}=X \times X \times X$ equipped with the norm $\|x\|_{X^{3}}=\left\|\left(x_{1}, x_{2}, x_{3}\right)\right\|_{X^{3}}=\left\|x_{1}\right\|+\left\|x_{2}\right\|+\left\|x_{3}\right\|$. Using the result of Lemma 2.3, we define the operator $\Psi: X^{3} \rightarrow X^{3}$ by

$$
\Psi x(t)=\left(\Psi_{1} x_{1}(t), \Psi_{2} x_{2}(t), \Psi_{3} x_{3}(t)\right)
$$

where

$$
\begin{aligned}
\Psi_{k} x_{k}(t)= & I_{0}^{\alpha_{k}} f_{k}(t, x(t))+\sum_{m=1}^{3}\left(d_{k, m} I_{0}^{\alpha_{m}} f_{m}(T, x(T))\right. \\
& \left.+\left(e_{k, m}+t b_{k, m}\right) I_{0}^{\alpha_{m}-1} f_{m}(T, x(T))\right) .
\end{aligned}
$$

If the operator $\Psi_{k}: X \rightarrow X$ given by (3.1) has a fixed point in $X$, then $\Psi_{k} x_{k}=x_{k}, k=1,2,3$. Hence in connection with Definition 2.5, we let $\Psi_{1} x_{1}=\digamma\left(x_{1}, x_{2}, x_{3}\right), \Psi_{2} x_{2}=\digamma\left(x_{2}, x_{1}, x_{3}\right)$, 
and $\Psi_{3} x_{3}=\digamma\left(x_{3}, x_{2}, x_{1}\right)$. This assumption connects the definition of the tripled fixed point introduced in Definition 2.5 with the fixed point of the tripled operator $\Psi=\left(\Psi_{1}, \Psi_{2}, \Psi_{3}\right)$. By this idea, we obtain the main results later.

We make use of the following assumption:

(A) Let $f_{k}: J \times X^{3} \rightarrow X, k=1,2,3$, be a jointly continuous function, and there exists a positive constant $L_{k}$ such that

$$
\left|f_{k}(t, x)-f_{k}(t, y)\right| \leq L_{k}\|x-y\|_{X^{3}}
$$

for all $t \in J$ and $x, y \in X^{3}$.

Theorem 3.1 Let condition ( $\Lambda$ ) be satisfied. Then tripled system (1.1) has a unique solution whenever

$$
\eta=\sum_{k=1}^{3}\left(\frac{L_{k} T^{\alpha_{k}}}{\Gamma\left(\alpha_{k}+1\right)}+\sum_{m=1}^{3} L_{m}\left(\left(\frac{\left|d_{k, m}\right|}{\alpha_{m}}+\left|b_{k, m}\right|\right) T+\left|e_{k, m}\right|\right) \frac{T^{\alpha_{m}-1}}{\Gamma\left(\alpha_{m}\right)}\right)<1 .
$$

Proof Let $B_{r}=\left\{x \in X^{3}:\|x\|_{X^{3}} \leq r\right\}$ be a closed subset in $X^{3}$ such that

$$
r>(1-\eta)^{-1} \sum_{k=1}^{3}\left(\frac{N_{k} T^{\alpha_{k}}}{\Gamma\left(\alpha_{k}+1\right)}+\sum_{m=1}^{3} N_{m}\left(\left(\frac{\left|d_{k, m}\right|}{\alpha_{m}}+\left|b_{k, m}\right|\right) T+\left|e_{k, m}\right|\right) \frac{T^{\alpha_{m}-1}}{\Gamma\left(\alpha_{m}\right)}\right) .
$$

Firstly, we show that $\Psi\left(B_{r}\right) \subset B_{r}$. For this, define $\sup _{t \in J}\left|f_{k}(t, 0)\right|=N_{k}<\infty, k=1,2,3$, then $\left|f_{k}(t, x)\right| \leq L_{k}\|x\|_{X^{3}}+N_{k}$ for any $t \in J$. Therefore

$$
\begin{aligned}
\left|\Psi_{k} x_{k}(t)\right| \leq & \frac{t^{\alpha_{k}}}{\Gamma\left(\alpha_{k}+1\right)}\left(L_{k}\|x\|_{X^{3}}+N_{k}\right) \\
& +\sum_{m=1}^{3}\left(\frac{\left|d_{k, m}\right| T^{\alpha_{m}}}{\Gamma\left(\alpha_{m}+1\right)}+\left(\left|e_{k, m}\right|+t\left|b_{k, m}\right|\right) \frac{T^{\alpha_{m}-1}}{\Gamma\left(\alpha_{m}\right)}\right)\left(L_{m}\|x\|_{X^{3}}+N_{m}\right) \\
\leq & \left(\frac{L_{k} t^{\alpha_{k}}}{\Gamma\left(\alpha_{k}+1\right)}+\sum_{m=1}^{3} L_{m}\left(\frac{\left|d_{k, m}\right| T}{\alpha_{m}}+\left(\left|e_{k, m}\right|+t\left|b_{k, m}\right|\right)\right) \frac{T^{\alpha_{m}-1}}{\Gamma\left(\alpha_{m}\right)}\right) r \\
& \times\left(\frac{N_{k} t^{\alpha_{k}}}{\Gamma\left(\alpha_{k}+1\right)}+\sum_{m=1}^{3} N_{m}\left(\frac{\left|d_{k, m}\right| T}{\alpha_{m}}+\left(\left|e_{k, m}\right|+t\left|b_{k, m}\right|\right)\right) \frac{T^{\alpha_{m}-1}}{\Gamma\left(\alpha_{m}\right)}\right) .
\end{aligned}
$$

Consequently,

$$
\begin{aligned}
\|\Psi x\|_{X^{3}} \leq & \sum_{k=1}^{3}\left(\frac{N_{k} T^{\alpha_{k}}}{\Gamma\left(\alpha_{k}+1\right)}+\sum_{m=1}^{3} N_{m}\left(\left(\frac{\left|d_{k, m}\right|}{\alpha_{m}}+\left|b_{k, m}\right|\right) T+\left|e_{k, m}\right|\right) \frac{T^{\alpha_{m}-1}}{\Gamma\left(\alpha_{m}\right)}\right) \\
& +\sum_{k=1}^{3}\left(\frac{L_{k} T^{\alpha_{k}}}{\Gamma\left(\alpha_{k}+1\right)}+\sum_{m=1}^{3} L_{m}\left(\left(\frac{\left|d_{k, m}\right|}{\alpha_{m}}+\left|b_{k, m}\right|\right) T+\left|e_{k, m}\right|\right) \frac{T^{\alpha_{m}-1}}{\Gamma\left(\alpha_{m}\right)}\right) r \\
\leq & r .
\end{aligned}
$$


Next, we show that the operator $\Psi$ is a contraction. For this, let $x, y \in X^{3}$, then for any $t \in J$ we get

$$
\begin{aligned}
& \left|\Psi_{k} x_{k}(t)-\Psi_{k} y_{k}(t)\right| \\
& \quad \leq\left(\frac{L_{k} t^{\alpha_{k}}}{\Gamma\left(\alpha_{k}+1\right)}+\sum_{m=1}^{3} L_{m}\left(\frac{\left|d_{k, m}\right| T}{\alpha_{m}}+\left(\left|e_{k, m}\right|+t\left|b_{k, m}\right|\right)\right) \frac{T^{\alpha_{m}-1}}{\Gamma\left(\alpha_{m}\right)}\right)\|x-y\|_{X^{3}} .
\end{aligned}
$$

It follows that

$$
\begin{aligned}
\|\Psi x-\Psi y\|_{X^{3}} & \leq \sum_{k=1}^{3}\left(\frac{L_{k} T^{\alpha_{k}}}{\Gamma\left(\alpha_{k}+1\right)}+\sum_{m=1}^{3} L_{m}\left(\left(\frac{\left|d_{k, m}\right|}{\alpha_{m}}+\left|b_{k, m}\right|\right) T+\left|e_{k, m}\right|\right) \frac{T^{\alpha_{m}-1}}{\Gamma\left(\alpha_{m}\right)}\right) \\
& \leq \eta\|x-y\|_{X^{3}} .
\end{aligned}
$$

Since $\eta<1$, therefore $\Psi$ is a contraction operator. Then, by the Banach fixed point theorem, the operator $\Psi$ has a unique fixed point which is the unique solution of problem (1.1). This completes the proof.

In the next result, we apply the Krasnoselskii fixed point theorem (Theorem 2.7) to prove the existence of at least one solution of the tripled fractional system (1.1). For this purpose, we decompose the triple operator $\Psi: X^{3} \rightarrow X^{3}$ into two triple operators $\Psi_{1}$ and $\Psi_{2}$ such that

$$
\Psi x(t)=\Psi_{1} x(t)+\Psi_{2} x(t)
$$

where $\Psi_{i} x(t)=\left(\Psi_{1, i} x_{1}(t), \Psi_{2, i} x_{2}(t), \Psi_{3, i} x_{3}(t)\right), i=1,2$, and

$$
\left\{\begin{array}{l}
\Psi_{k, 1} x_{k}(t)=I_{0}^{\alpha_{k}} f_{k}(t, x(t)), \quad k=1,2,3, \\
\Psi_{k, 2} x_{k}(t)=\sum_{m=1}^{3}\left(d_{k, m} I_{0}^{\alpha_{m}} f_{m}(T, x(T))+\left(e_{k, m}+t b_{k, m}\right) I_{0}^{\alpha_{m}-1} f_{m}(T, x(T))\right) .
\end{array}\right.
$$

Theorem 3.2 Let $f_{k}: J \times X^{3} \rightarrow X, k=1,2,3$, be a jointly continuous function, and there exist nonnegative fractional integrable real-valued functions $\varphi_{k}$ and $\mu_{k}$ such that

$$
\left\{\begin{array}{l}
\left|f_{k}(t, x)-f_{k}(t, y)\right| \leq \varphi_{k}(t)\|x-y\|_{X^{3}} \\
\left|f_{k}(t, 0)\right| \leq \mu_{k}(t), \quad t \in J, k=1,2,3
\end{array}\right.
$$

where $x, y \in X^{3}$. Then tripled system (1.1) has a solution provided that

$$
\sum_{k=1}^{3} m_{k} \max _{t \in J} I_{0}^{\alpha_{k}} \varphi_{k}(t)+n_{k} \max _{t \in J} I_{0}^{\alpha_{k}-1} \varphi_{k}(t)<1,
$$

where $m_{k}=1+\sum_{m=1}^{3}\left|d_{m, k}\right|$ and $n_{k}=\sum_{m=1}^{3}\left|e_{m, k}\right|+T\left|b_{m, k}\right|$.

Proof Let $B_{r}=\left\{x \in X^{3}:\|x\|_{X^{3}} \leq r\right\}$ be a closed convex nonempty subset in $X^{3}$ such that

$$
r \geq \frac{\sum_{k=1}^{3} m_{k} \max _{t \in J} I_{0}^{\alpha_{k}} \mu_{k}(t)+n_{k} \max _{t \in J} I_{0}^{\alpha_{k}-1} \mu_{k}(t)}{1-\sum_{k=1}^{3} m_{k} \max _{t \in J} I_{0}^{\alpha_{k}} \varphi_{k}(t)+n_{k} \max _{t \in J} I_{0}^{\alpha_{k}-1} \varphi_{k}(t)} .
$$


We show that $\Psi_{1}$ is a contraction and $\Psi_{2}$ is compact on $B_{r}$. Before doing these two steps, we show that $\Psi_{1} x+\Psi_{2} y \in B_{r}$ whenever $x, y \in B_{r}$. Let $x=\left(x_{1}, x_{2}, x_{3}\right)$ and $y=\left(y_{1}, y_{2}, y_{3}\right)$ be any elements of $B_{r}$, then for $t \in J$ we have

$$
\left|\Psi_{k, 1} x_{k}(t)\right| \leq I_{0}^{\alpha_{k}}\left|f_{k}(t, x(t))\right| \leq I_{0}^{\alpha_{k}} \varphi_{k}(t)\|x\|_{X^{3}}+I_{0}^{\alpha_{k}} \mu_{k}(t),
$$

and

$$
\begin{aligned}
\left|\Psi_{k, 2} y_{k}(t)\right| \leq & \sum_{m=1}^{3}\left(\left|d_{k, m}\right| I_{0}^{\alpha_{m}}\left|f_{m}(T, y(T))\right|+\left(\left|e_{k, m}\right|+t\left|b_{k, m}\right|\right) I_{0}^{\alpha_{m}-1}\left|f_{m}(T, y(T))\right|\right) \\
\leq & \|y\|_{X^{3}} \sum_{m=1}^{3}\left|d_{k, m}\right| I_{0}^{\alpha_{m}} \varphi_{m}(t)+\left(\left|e_{k, m}\right|+t\left|b_{k, m}\right|\right) I_{0}^{\alpha_{m}-1} \varphi_{m}(t) \\
& +\sum_{m=1}^{3}\left|d_{k, m}\right| I_{0}^{\alpha_{m}} \mu_{m}(t)+\left(\left|e_{k, m}\right|+t\left|b_{k, m}\right|\right) I_{0}^{\alpha_{m}-1} \mu_{m}(t) .
\end{aligned}
$$

In consequence, we obtain

$$
\left\|\Psi_{1} x\right\|_{X^{3}} \leq\|x\|_{X^{3}} \sum_{k=1}^{3} \max _{t \in J} I_{0}^{\alpha_{k}} \varphi_{k}(t)+\sum_{k=1}^{3} \max _{t \in J} I_{0}^{\alpha_{k}} \mu_{k}(t)
$$

and

$$
\begin{aligned}
\left\|\Psi_{2} y\right\|_{X^{3}} \leq & \|y\|_{X^{3}} \sum_{m=1}^{3} \sum_{k=1}^{3}\left|d_{k, m}\right| \max _{t \in J} I_{0}^{\alpha_{m}} \varphi_{m}(t)+\left(\left|e_{k, m}\right|+T\left|b_{k, m}\right|\right) \max _{t \in J} I_{0}^{\alpha_{m}-1} \varphi_{m}(t) \\
& +\sum_{m=1}^{3}\left|d_{k, m}\right| \max _{t \in J} I_{0}^{\alpha_{m}} \mu_{m}(t)+\left(\left|e_{k, m}\right|+t\left|b_{k, m}\right|\right) \max _{t \in J} I_{0}^{\alpha_{m}-1} \mu_{m}(t) .
\end{aligned}
$$

Hence

$$
\begin{aligned}
\left\|\Psi_{1} x+\Psi_{2} y\right\|_{X^{3}} \leq & r \sum_{k=1}^{3} m_{k} \max _{t \in J} I_{0}^{\alpha_{k}} \varphi_{k}(t)+n_{k} \max _{t \in J} I_{0}^{\alpha_{k}-1} \varphi_{k}(t) \\
& +\sum_{k=1}^{3} m_{k} \max _{t \in J} I_{0}^{\alpha_{k}} \mu_{k}(t)+n_{k} \max _{t \in J} I_{0}^{\alpha_{k}-1} \mu_{k}(t) .
\end{aligned}
$$

In accordance with the previous estimates and the value of $r$, we deduce that $\Psi_{1} x+\Psi_{2} y \in$ $B_{r}$.

Next we show the contraction of $\Psi_{1}$. Let $x, y \in X^{3}$, then

$$
\begin{aligned}
\left|\Psi_{k, 1} x_{k}(t)-\Psi_{k, 1} y_{k}(t)\right| & \leq I_{0}^{\alpha_{k}}\left|f_{k}(t, x(t))-f_{k}(t, y(t))\right| \\
& \leq I_{0}^{\alpha_{k}} \varphi_{k}(t)\|x-y\|_{X^{3}} \\
& \leq \max _{t \in J} I_{0}^{\alpha_{k}} \varphi_{k}(t)\|x-y\|_{X^{3}} .
\end{aligned}
$$


Hence

$$
\left\|\Psi_{1} x-\Psi_{1} y\right\|_{X^{3}} \leq\left(\sum_{k=1}^{3} \max _{t \in J} I_{0}^{\alpha_{k}} \varphi_{k}(t)\right)\|x-y\|_{X^{3}} .
$$

Since $\max _{t \in J} I_{0}^{\alpha_{k}} \varphi_{k}(t) \leq m_{k} \max _{t \in J} I_{0}^{\alpha_{k}} \varphi_{k}(t)<1$, we deduce the contraction.

The last step shows the compactness of $\Psi_{2}$. It is obvious by (3.2) that $\Psi_{2}$ maps bounded sets into bounded sets. On the other hand, the continuity of $f_{k}$ and its fractional integral would imply the continuity of the operator $\Psi_{2}$. The only thing we add is the equicontinuity of the family $\Psi_{2} B_{r}$. Let $t_{1}, t_{2} \in J$ with $t_{1}<t_{2}$, then we have

$$
\left|\Psi_{k, 2} x_{k}\left(t_{2}\right)-\Psi_{k, 2} x_{k}\left(t_{1}\right)\right| \leq\left(t_{2}-t_{1}\right) \sum_{m=1}^{3}\left|b_{k, m}\right|\left(I_{0}^{\alpha_{m}-1}\left(r \varphi_{m}(T)+\mu_{m}(T)\right)\right) .
$$

Accordingly, we find that

$$
\left\|\Psi_{2} x\right\|_{X^{3}} \leq\left(t_{2}-t_{1}\right) \sum_{m=1}^{3}\left(\left(I_{0}^{\alpha_{m}-1}\left(r \varphi_{m}(T)+\mu_{m}(T)\right)\right) \sum_{m=1}^{3}\left|b_{k, m}\right|\right)
$$

which tends to zero as $t_{1} \rightarrow t_{2}$ independently of $x$. Hence, by the Arzelà-Ascoli theorem, the operator $\Psi_{2}$ is compact. Using Krasnoselskii Theorem 2.7, there exists a fixed point $x \in B_{r} \subset X^{3}$ satisfying the operator equation $x=\Psi_{1} x+\Psi_{2} x$, which is the solution of tripled system (1.1). This completes the proof.

\section{Application}

Corresponding to system (1.1), we consider the following tripled fractional system:

$$
\left\{\begin{array}{l}
{ }^{c} D_{0}^{\frac{6}{5}} x_{1}(t)=\frac{t}{20}+\frac{1}{\sqrt{169+t^{2}}}\left(\frac{\left|x_{1}(t)\right|}{1+|x(t)|}+\frac{\left|x_{2}(t)\right|}{1+|y(t)|}+\frac{\left|x_{3}(t)\right|}{1+|z(t)|}\right), \\
{ }^{c} D_{0}^{\frac{3}{2}} x_{2}(t)=\frac{1}{\sqrt{64+t^{2}}}+\frac{t\left|x_{1}(t)\right|}{20}+\frac{t\left|x_{2}(t)\right|}{15\left(t^{3}+1\right)}+\frac{t\left|x_{3}(t)\right|}{15(2+t)}, \\
{ }^{c} D_{0}^{\frac{9}{8}} x_{3}(t)=\frac{e^{-t}}{10+t}+\frac{\left|x_{1}(t)\right|}{\sqrt{169+t^{2}}}+\frac{\left|x_{2}(t)\right|}{12+t}+\frac{\left|x_{3}(t)\right|}{15 \sqrt{1+t^{2}}},
\end{array}\right.
$$

where $t \in[0,1]$ with

$$
\left\{\begin{array}{l}
x_{1}(0)=x_{2}(1), \quad 5 x_{1}^{\prime}(0)=2 x_{2}^{\prime}(1) \\
2 x_{2}(0)=x_{3}(1),
\end{array}\right.
$$

Using the given data, we find the following constants:

$$
\begin{aligned}
& a_{1,0}=1, \quad a_{1,1}=\frac{2}{5}, \quad a_{2,0}=\frac{1}{2}, \quad a_{2,1}=\frac{2}{7}, \\
& a_{3,0}=\frac{1}{3}, \quad a_{3,1}=\frac{3}{4}, \\
& b_{1,1}=b_{2,2}=b_{3,3}=0.09, \quad b_{1,2}=0.4375, \quad b_{1,3}=0.125, \\
& b_{2,1}=0.2344, \quad b_{2,3}=0.3125, \quad b_{3,1}=0.8203, \quad b_{3,2}=0.3281,
\end{aligned}
$$




$$
\begin{aligned}
& d_{1,1}=d_{2,1}=d_{2,2}=d_{3,3}=0.2, \quad d_{1,2}=1.2, \quad d_{1,3}=d_{2,3}=0.6, \\
& d_{3,1}=0.4=d_{3,2}, \quad e_{1,1}=0.7921, \quad e_{1,2}=0.397, \quad e_{1,3}=0.4562, \\
& e_{2,1}=0.5578, \quad e_{2,2}=0.3031, \quad e_{2,3}=0.1437, \\
& e_{3,1}=0.2953, \quad e_{3,2}=0.2781, \quad e_{3,3}=0.1937, \\
& L_{1}=\frac{1}{13}, \quad L_{2}=\frac{1}{15}, \quad L_{3}=\frac{1}{12} .
\end{aligned}
$$

Then we deduce that $\eta \approx 0.95<1$. Hence by Theorem 3.1 there is a unique solution for system (4.1). Furthermore, we have

$$
\begin{array}{lll}
\varphi_{1}(t)=\frac{1}{\sqrt{169+t^{2}}}, & \varphi_{2}(t)=\frac{t}{15}, & \varphi_{3}(t)=\frac{1}{12}, \\
\mu_{1}(t)=\frac{t}{20}, & \mu_{2}(t)=\frac{1}{\sqrt{64+t^{2}}}, & \mu_{3}(t)=\frac{e^{-t}}{10+t},
\end{array}
$$

and

$$
\begin{aligned}
& m_{1}=1.8, \quad m_{2}=2.8, \quad m_{3}=2.4, \\
& n_{1}=2.79, \quad n_{2}=1.834, \quad n_{3}=1.3211 .
\end{aligned}
$$

Hence

$$
\sum_{k=1}^{3} m_{k} \max _{t \in J} I_{0}^{\alpha_{k}} \varphi_{k}(t)+n_{k} \max _{t \in J} I_{0}^{\alpha_{k}-1} \varphi_{k}(t)=0.812<1 .
$$

Therefore, using Theorem 3.2, there exists a solution of system (4.1). The reduction of the condition value from 0.95 to 0.812 is substantial. However, we lose the uniqueness property of the solution.

The used permutation in the boundary condition (4.2) has the form (1 23 ). Let us use another permutation of the boundary conditions for system (4.1) that has the form (13)(2) such that

$$
\left\{\begin{array}{l}
x_{1}(0)=x_{3}(1), \quad 5 x_{1}^{\prime}(0)=2 x_{3}^{\prime}(1), \\
2 x_{2}(0)=x_{2}(1), \quad 7 x_{2}^{\prime}(0)=2 x_{2}^{\prime}(1), \\
3 x_{3}(0)=x_{1}(1), \quad 4 x_{3}^{\prime}(0)=3 x_{1}^{\prime}(1)
\end{array}\right.
$$

with the same constants as (4.3). Hence we deduce the same results as in the previous example. Furthermore, one can use four other permutations, namely (1 2)(3), (1)(2 3), (1 3 2), and identity (1)(2)(3).

\section{Conclusion}

In this paper, we investigate a tripled system of three fractional differential equations of or$\operatorname{der} \alpha \in(1,2]$. The existence and uniqueness of solutions of the proposed system associated with cyclic permutation boundary conditions are established. The Banach and Krasnoselskii fixed point theorems are used as tools to prove our main results. We present examples to illustrate the applicability of the main results. 
We study a fractional system consisting of three associated equations together with a new type of boundary conditions that is related to permutation groups. This might be a novel approach that will provide substantial potential for developing more new ideas in this field.

The results of this paper can be extended to a tripled system of fractional equations with impulsive effects and nonlocal conditions. Indeed, a tripled fractional system along with different boundary conditions can be considered and discussed. Finally, the results of this paper can be extended to $m$-tuple fractional systems. We leave investigation of these topics as future work for interested readers.

\section{Acknowledgements}

The authors would like to thank the referees for their valuable comments and suggestions which helped in improving the contents of the paper.

\section{Funding}

The third author would like to thank Prince Sultan University for supporting this work through research group Nonlinear Analysis Methods in Applied Mathematics (NAMAM) group number RG-DES-2017-01-17.

Availability of data and materials

Not applicable.

\section{Competing interests}

On behalf of all authors, the corresponding author declares that there are no competing interests in this paper.

\section{Authors' contributions}

The authors contributed equally and significantly to the contents of the paper. All authors read and approved the final manuscript.

\section{Author details}

'Department of Mathematics, Al-Azhar University-Gaza, Gaza, Palestine. ²Department of Mathematics and Genera Sciences, Prince Sultan University, Riyadh, 11586, Saudi Arabia.

\section{Publisher's Note}

Springer Nature remains neutral with regard to jurisdictional claims in published maps and institutional affiliations.

Received: 22 July 2019 Accepted: 9 August 2020 Published online: 18 August 2020

\section{References}

1. Abdalla, B., Abodayeh, K., Abdeljawad, T., Alzabut, J.: New oscillation criteria for forced nonlinear fractional difference equations. Vietnam J. Math. 2017(45), 609-618 (2017)

2. Abdeljawad, T., Alzabut, J.: On Riemann-Liouville fractional $q$-difference equations and its application to retarded logistic type model. Math. Methods Appl. Sci. 41, 8953-8962 (2018)

3. Abdeljawad, T., Jarad, F., Alzabut, J.: Fractional proportional differences with memory. Eur. Phys. J. Spec. Top. 226, 3333-3354 (2017)

4. Ahmad, M., Zada, A., Alzabut, J.: Hyres-Ulam stability of coupled system of fractional differential equations of Hilfer-Hadamard type. Demonstr. Math. 2019(52), 283-295 (2019)

5. Alzabut, J., Abdeljawad, T., Baleanu, D.: Nonlinear delay fractional difference equations with applications on discrete fractional Lotka-Volterra competition model. J. Comput. Anal. Appl. 25(5), 889-898 (2018)

6. Ahmad, B., Matar, M.M., El-Salmy, O.M.: Existence of solutions and Ulam stability for Caputo type sequential fractional differential equations of order $\alpha \in(2,3)$. Int. J. Anal. Appl. 15, 86-101 (2017)

7. Ahmad, B., Ntouyas, S.K., Alsaedi, A.: On fully coupled nonlocal multi-point boundary value problems of nonlinear mixed-order fractional differential equations on an arbitrary domain. Filomat 2018(32), 13 (2018)

8. Ahmad, B., Ntouyas, S., Alsaedi, A.: Fractional order differential systems involving right Caputo and left Riemann-Liouville fractional derivatives with nonlocal coupled conditions. Bound. Value Probl. 2019, Article ID 109 (2019)

9. Alzabut, J., Tyagi, S., Abbas, S.: Discrete fractional order BAM neural networks with leakage delay: existence and stability results. Asian J. Control 22(1), 1-13 (2020)

10. Baeza, L., Ouyang, H.: A railway track dynamics model based on modal substructuring and a cyclic boundary condition. J. Sound Vib. 330(1), 75-86 (2011)

11. Balachandran, K., Matar, M.M., Trujilo, J.J.: Note on controllability of linear fractional dynamical system. J. Control Decis. 3(4), 267-279 (2015)

12. Berinde, V., Borcut, M.: Tripled fixed point theorems for contractive type mappings in partially ordered metric spaces. Nonlinear Anal., Theory Methods Appl. 74(15), 4889-4897 (2011)

13. Chadha, A., Sakthivel, R., Bora, S.N.: Solvability of control problem for fractional nonlinear differential inclusions with nonlocal conditions. Nonlinear Anal., Model. Control 24, 503-522 (2019) 
14. Chen, Y., An, H.: Numerical solutions of coupled Burgers equations with time and space fractional derivatives. Appl. Math. Comput. 200, 87-95 (2008)

15. Cui, M., Zhu, Y., Pang, H.: Existence and uniqueness results for a coupled fractional order systems with the multi-strip and multi-point mixed boundary conditions. Adv. Differ. Equ. 2017(2017), 224 (2017)

16. Ding, Y., Li, Y.: Monotone iterative technique for periodic problem involving Riemann-Liouville fractional derivatives in Banach spaces. Bound. Value Probl. 2018, Article ID 119 (2018)

17. Diop, M.A., Rathinasamy, S., Ndiaye, A.A.: Neutral stochastic integrodifferential equations driven by a fractional Brownian motion with impulsive effects and time-varying delays. Mediterr. J. Math. 13(5), 2425-2442 (2016)

18. Ertürk, V.S., Momani, S.: Solving systems of fractional differential equations using differential transform method. J. Comput. Appl. Math. 215, 142-151 (2008)

19. Fraleigh, J.B.: A First Course in Abstract Algebra. Pearson, England (2014)

20. Granas, A., Dugundji, J.: Fixed Point Theory. Springer, New York (2005)

21. Henderson, J., Luca, R.A.: On a system of fractional differential equations with coupled integral boundary conditions. Fract. Calc. Appl. Anal. 18, 361-386 (2015)

22. Jafari, H., Daftardar-Gejji, V:: Solving a system of nonlinear fractional differential equations using Adomian decomposition. J. Comput. Appl. Math. 196(2), 644-651 (2006)

23. Goubet, O., Manoubi, I.: Theoretical analysis of a water wave model with a nonlocal viscous dispersive term using the diffusive approach. Adv. Nonlinear Anal. 8(1), 253-266 (2019)

24. Karakaya, K., Bouzara, N.-E., DoLan, K., Atalan, Y.: Existence of tripled fixed points for a class of condensing operators in Banach spaces. Sci. World J. 2014, Article ID 541862 (2014). https://doi.org/10.1155/2014/541862

25. Kilbas, A.A., Srivastava, H.M., Trujillo, J.J.: Theory and Applications of Fractional Differential Equations. Elsevier, Amsterdam (2006)

26. Liu, Y:: A new method for converting boundary value problems for impulsive fractional differential equations to integral equations and its applications. Adv. Nonlinear Anal. 2019(1), 386-454 (2019)

27. Magin, R.L.: Fractional Calculus in Bioengineering. Begell, House Publisher, Connecticut (2006)

28. Matar, M.M.: Controllability of fractional semilinear mixed Volterra-Fredholm integrodifferential equations with nonlocal conditions. Int. J. Math. Anal. 4(23), 1105-1116 (2010)

29. Matar, M.M.: On controllability of linear and nonlinear fractional integrodifferential systems. Fract. Differ. Calc. 9(1), 19-32 (2019)

30. Matar, M.M., Abo Amra, I.E.: Existence of mild solutions for non-periodic coupled fractional differential equations. J. Fract. Calc. Appl. 11(1), 41-53 (2020)

31. Matar, M.M., Abu Ghalwa, H.N.: Approximate controllability of nonlocal fractional integrodifferential control systems of order $1<\alpha<2$. Acta Math. Univ. Comen. 88(1), 131-144 (2019)

32. Matar, M.M., Abu Skhail, E.S.: On stability of nonautonomous perturbed semilinear fractional differential systems of order $\alpha \in(1,2)$. J. Math. 2018, Article ID 1723481 (2018). https://doi.org/10.1155/2018/1723481

33. Momani, S., Qaralleh, R.: An efficient method for solving systems of fractional integro-differential equations. Comput. Math. Appl. 52, 459-470 (2006)

34. Podlubny, I.: Fractional Differential Equations. Academic Press, San Diego (1999)

35. Pratap, A., Raja, R., Alzabut, J., Dianavinnarasi, J., Cao, J., Rajchakit, G.: Finite-time Mittag-Leffler stability of fractional-order quaternion-valued memristive neural networks with impulses. Neural Process. Lett. (2019). https://doi.org/10.1007/s11063-019-10154-1

36. Samko, S.G., Kilbas, A.A., Marichev, O.I.: Fractional Integrals and Derivatives: Theory and Applications. Gordon \& Breach, Yverdon (1993)

37. Xu, N., Liu, W.: Iterative solutions for a coupled system of fractional differential-integral equations with two-point boundary conditions. Appl. Math. Comput. 244, 903-911 (2014)

38. Zada, A., Waheed, H., Alzabut, J., Wang, X.: Existence and stability of impulsive coupled system of fractional integrodifferential equations. Demonstr. Math. 2019(52), 296-335 (2019)

39. Zhang, Y.: Existence results for a coupled system of nonlinear fractional multi-point boundary value problems at resonance. J. Inequal. Appl. 2018, 198 (2018)

\section{Submit your manuscript to a SpringerOpen ${ }^{\circ}$ journal and benefit from:}

- Convenient online submission

- Rigorous peer review

- Open access: articles freely available online

- High visibility within the field

- Retaining the copyright to your article

Submit your next manuscript at $\gg$ springeropen.com 